\title{
Harmonic Mitigation Potential of Shunt Harmonic Impedances
}

\author{
Wouter Ryckaert, member, IEEE, Jozef A.L. Ghijselen, member, IEEE, \\ and Jan A.A. Melkebeek, senior member, IEEE
}

\begin{abstract}
The amount of non-linear and unbalanced loads connected to the electric power system is steadily growing. The power quality (i.e. the reliability of the energy supply and the quality of the voltage waveform) may be severely degraded, causing many unfavourable effects on the power system and the connected loads. Especially in the case of resonances, there can be a severe voltage distortion. In case of a resonance due to existing capacitor banks or cables, a damping technique is proposed through the application of resistive shunt harmonic impedances (SHI). These impedances can be implemented using active power electronic devices that synthesize a resistive load. Benefits : no need to measure the current, improved stability margin, ability to be effective for all harmonics
\end{abstract}

Index Terms-Electric Power Systems, Power Quality, Nonlinear Loads

\section{INTRODUCTION}

$\mathbf{S}$ INCE the early 1970's, with the advent of power electronic devices in rectifiers, motor drives, and power supplies, the growing levels of harmonics in electric power systems has worried power system engineers. In the ideal case, the voltage of the electric power system is sinusoidal and symmetric. The exploding use of personal computers and electric loads controlled by power electronics has led to a severe increase of current harmonics drawn from the distribution line. Due to the impedance of the distribution line, the harmonic currents lead to harmonic voltage distortion. The harmonic currents cause many unfavourable effects on the power system itself and the loads connected with it [1]. Some of the unfavourable effects are: overheating of transformers and wiring, damaging of capacitor banks, malfunctioning of electronic equipment,... Because of these drawbacks, the interest in harmonic distortion has increased for both manufacturers of electronic equipment and the utilities. Both the quality of the mains voltage wave shape and the reliability of the energy distribution constitute the so-called power quality. The increasing amount of non-linear loads will increase the problems concerning the harmonic pollution and in the future, research will become more important.

\section{HARMONIC MitigATION TECHNIQUES}

In the last two decades, power quality improvement has become an important target for the product developer and the energy provider. To meet the existing standards, one

Wouter Ryckaert is with the Department of Electrical Energy, Systems and Automation (EESA), Electrical Energy Laboratory (EELAB), Ghen University (UGent), Sint-Pietersnieuwstraat 41, B-9000 Gent, Belgium. Email: Wouter.Ryckaert@UGent.be . can install passive or active filters. It was proven that active filters are very effective to compensate harmonic currents and voltages [2]. As compared to passive filters, they do not have problems as resonance generation, component tuning and network impedance dependence. However, they are still very expensive. Therefore, active filters are only installed near large concentrations of polluting loads, e.g. industrial plants. It was suggested by Akagi [3] that shunt active filters, behaving as linear resistive impedances for harmonics, could be spread throughout the power system to reduce the propagation of harmonic pollution. Although complete compensation is only possible using true active filters, shunt harmonic resistances have been shown to provide a considerable reduction of the harmonic propagation. The advantage of this approach is the simplicity of the compensator devices because there is no need for the measurement of the polluting currents [4]. There is also an improved stability margin of the convertor as compared to active filters compensating for measured current pollution. This resistive impedance function can be realised as a secondary control function of power electronic energy supplies [5].

It has to be emphasized that the resistive shunt harmonic impedance is not a real resistance. It is a convertor, build with active devices, with a resistive behaviour for harmonics in steady state, which means that the equivalent load impedance $Z_{\text {load }}(h)=V_{\text {load }}(h) / I_{\text {load }}(h)$ is resistive for each harmonic order $h$ in steady state. The absorbed harmonic active power can be converted to fundamental power or can be used to compensate the switching losses. In this way, the absorbed harmonic active power is usefully applied and can not be seen as (ohmic) losses in a real resistance.

Because the simplicity of the control strategy, many power electronic loads can be equipped with the resistive behaviour for harmonics and can be spread throughout the distribution system. In this way, every optimised load has a contribution to the reduction of the harmonic pollution which is very important in the case of resonance conditions.

\section{Preferked harmonic impedance Behaviour}

In the first step of the research, the authors have shown that the preferred SHI to damp the harmonic propagation is resistive for all harmonics, with a magnitude of about $0.5-1 \mathrm{pu}$ (referred to the apparent power of the distribution transformer). Lower SHI values than 0.5 pu are not encouraged to prohibit large harmonic line currents to flow when the mains voltage 
is polluted [6].

It has been shown that it is not sufficient to realize an impedance for a limited number of harmonics [7]. For instance, tackling the strongest or one of the strongest harmonic voltage component(s) is not as effective because other harmonic components may be amplified because of the non-linear behaviour of the polluting loads.

The mitigation potential of a resistive SHI together with the mitigation potential of a SHI tackling only one of the strongest harmonic components is explained more in detail in the following paragraphs.

\section{A. System Description}

In Fig. 1 the equivalent circuit of a simple electric power system is shown. The mains voltage source is represented by

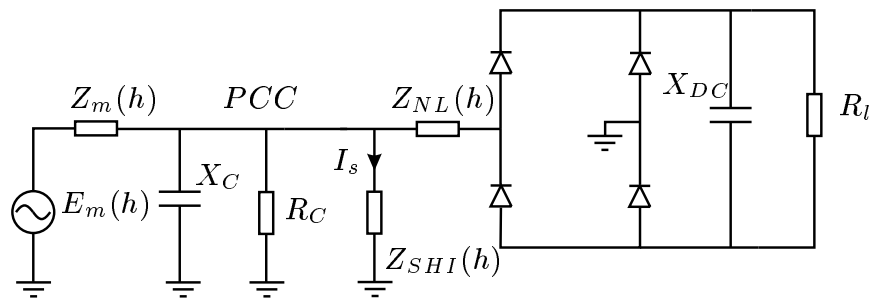

Fig. 1. Simple electric power system (with resonance(s)) containing a shunt harmonic impedance

its no-load voltage $E_{m}(h)$ and its internal mains impedance $Z_{m}(h)$. The mains impedance is usually dominated by the short-circuit impedance of the distribution transformer and the impedance of the connecting busbars and cables and is therefore resistive-inductive. The polluting load is a capacitively smoothed rectifier. The rectifier filter impedance $Z_{N L}$ may serve to model both the actual filter impedance of the rectifier and/or the local power impedance (e.g. caused by the cables). The capacitor $X_{c}$, e.g. used for power factor correction, is tuned to obtain a resonance at the 11 th harmonic order.

The parameters, referred to the apparent power of the distribution transformer, are: $\left|Z_{m}(1)\right|=0.06 p u, \angle Z_{m}(1)=\frac{\pi}{4}$, $\left|Z_{N L}(1)\right|=0.02 p u, \angle Z_{N L}(1)=\frac{\pi}{4}, X_{D C}(1)=0.12 p u$ and $R_{l}$ is adjusted to obtain an active power load of $P=\frac{S_{r e f}}{2}$. The capacitor generates $0.195 \mathrm{pu}$ of reactive power and its resistive loss is modelled as a parallel resistance $R_{c}$, drawing $0.0195 \mathrm{pu}$ of active power.

A SHI, represented by $Z_{S H I}(h)$ in steady state, is placed at the PCC to damp the harmonic mitigation.

\section{B. Resistive Shunt Harmonic Impedances}

To mitigate the voltage distortion at the PCC, one can install a SHI. In this section, the mitigation potential of a resistive SHI is investigated $Z_{S H I}(h)=R_{s}$. The results are shown in Table I. It follows that resistive shunt impedances are attractive to damp the resonance, certainly for values between $0.5 \mathrm{pu}$ and $1 \mathrm{pu}$ as the resulting $T H D_{v}$ value is reduced to about the value $(7.57 \%)$ of the system without resonance and $Z_{S H I}(h)=\infty$. As already mentioned, lower values of $R_{s}$ are not encouraged to prohibit excessive harmonic line currents when the mains voltage is polluted.
TABLE I

PCC VOLTAGE THD AND RMS VALUES OF $I_{s}$ FOR A TRUE RESISTIVE SHUNT HARMONIC IMPEDANCE (SYSTEM WITH RESONANCE)

\begin{tabular}{|c|c|c|}
\hline \hline$R_{s}(p u)$ & $\begin{array}{c}\text { THD } \\
\text { @ PCC }\end{array}$ & $\begin{array}{c}\text { Change } \\
\text { TH } D_{V}\end{array}$ \\
\hline \hline$\infty, X_{C}=\infty$ & 7.57 & \\
$\infty$ & 11.42 & Ref. \\
3 & 9.58 & $-16.1 \%$ \\
1 & 8.14 & $-28.7 \%$ \\
0.5 & 7.12 & $-37.7 \%$ \\
0.4 & 6.77 & $-40.7 \%$ \\
0.3 & 6.28 & $-45.0 \%$ \\
0.2 & 5.50 & $-51.8 \%$ \\
0.1 & 4.01 & $-64.9 \%$ \\
\hline
\end{tabular}

\section{A Single Harmonic Impedance}

To damp the harmonic propagation, the use of a single harmonic impedance is investigated, i.e. an impedance drawing current at a single harmonic order. In this case, the SHI is not restricted to resistive values. To limit the length of the paper and to cover the whole research topic, only one example is added. Other shunt harmonic impedance control strategies are mentioned in [7].

In Fig. 2, the relative change of the PCC voltage THD is shown for a SHI drawing current of the 5th harmonic order only. In the case of a resonance, creating a short-circuit for the strongest or one of the strongest harmonic components is not (always) a good solution. Figure 2 shows that, in this specific case, the PCC voltage THD slightly deteriorates when the 5 th harmonic component is short circuited $\left(\operatorname{Re}\left(Z_{S H I}(5)\right)=\right.$ $\left.\operatorname{Im}\left(Z_{S H I}(5)\right)=0\right)$. The reason is that other harmonic voltage

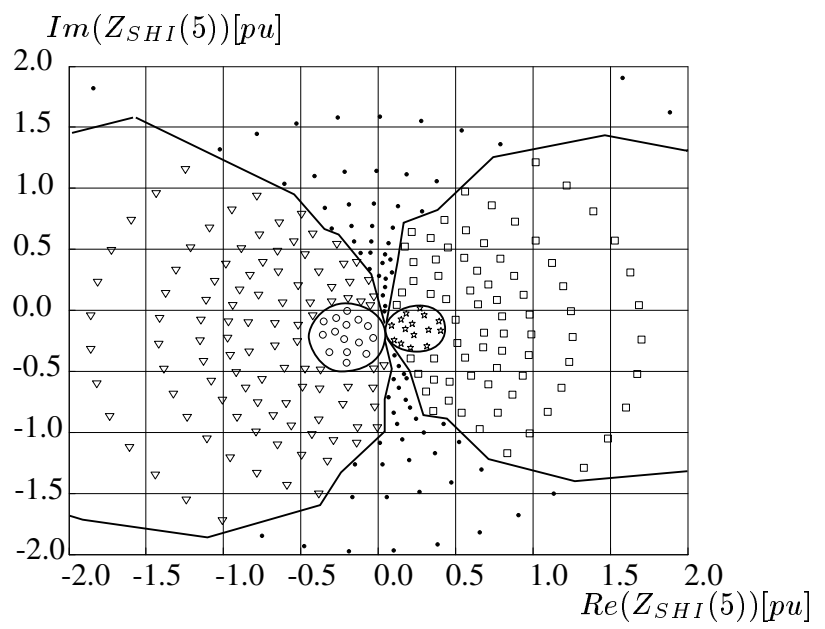

Fig. 2. PCC voltage THD increase for different values of $Z_{S H I}(5)$ : o: $>1.2, \nabla: 1.02 \ldots 1.2, \bullet: 0.98 \ldots 1.02, \quad: 0.9 \ldots 0.98, \quad:<0.9$

components are amplified through the commutation behaviour of the rectifier and through the remaining resonance(s). This is illustrated in Fig. 3, where the 5th harmonic component is 0 but other components are amplified.

Favourable values of $Z_{S H I}(h)=Z_{S H I}(5)$ are rather resistivecapacitive (Fig. 2). The minimum THD value which can be 


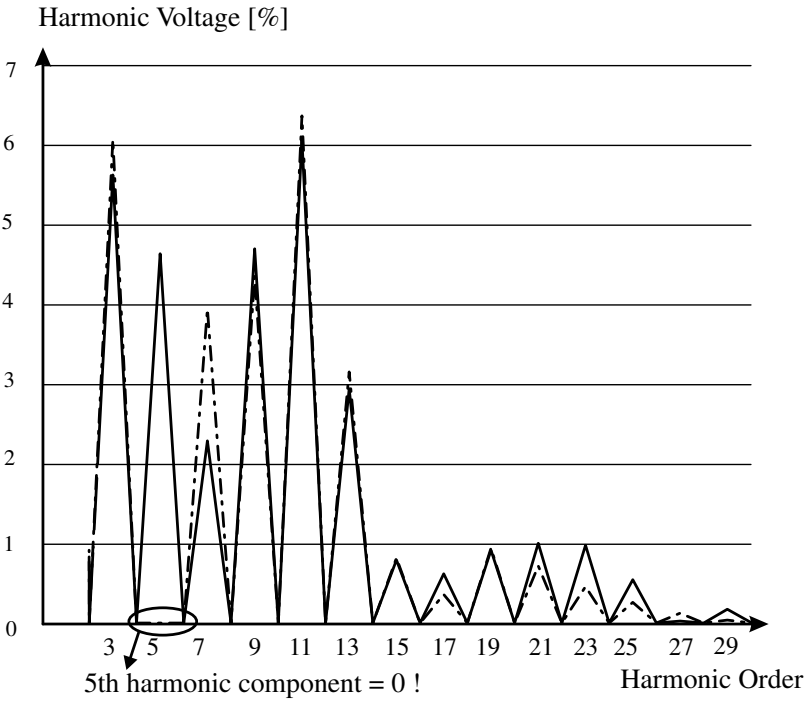

Fig. 3. PCC voltage spectrum for $Z_{S H I}(h)=Z_{S H I}(5)=0$ (dashed line) and for $Z_{S H I}(h)=\infty$ (solid line)

achieved with a 5th harmonic shunt impedance is $9.49 \%$ (reduction of $17 \%$ ) with $Z_{S H I}(5)=0.11-0.18 i \mathrm{pu}$.

\section{THE PLACEMENT OF A RESISTIVE SHI ALONG A DISTRIBUTION FEEDER}

The study in [7] discussed the favourable influence of a resistive SHI on the local voltage THD. In this section, the harmonic mitigation potential of a resistive SHI will be discussed together with its influence on the voltage THD along a typical distribution feeder, with the location of the SHI along the feeder as a parameter.

\section{A. Capacitor banks and non-linear loads concentrated in single nodes}

It is assumed that both the non-linear loads and the power factor correction capacitors are concentrated in single nodes (Fig. 4). The present study was realised for a generalized distribution system model (Fig. 4). The distribution system is

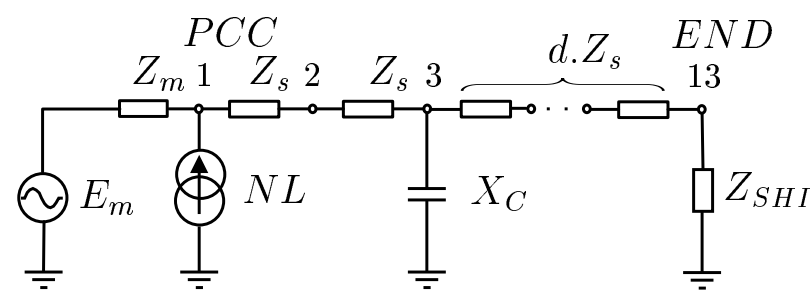

Fig. 4. Radial distribution feeder

fed at the Point of Common Coupling (PCC) by a distribution transformer, feeding a radial feeder branch containing 13 nodes. The parameters of the distribution system are chosen to match typical values found in practice:

- The distribution transformer $Z_{m}$ has a short-circuit impedance of $10 \%$ (referred to the total rating of the distribution system), and a phase angle of 80 degrees (inductive). These parameters are typical for large HV/MV distribution transformers;
- The impedance of the distribution feeder sections between the feeder nodes $Z_{s}$ is chosen to obtain a total (fundamental) voltage drop along the feeder of $0.12 \mathrm{pu}$ when the feeder is fully loaded, and the load is symmetrically and equally divided among the feeder nodes. The fundamental displacement factor of the loads equals 0.95 (inductive). It is assumed that the HV/MV transformer regulates the PCC voltage to $1.06 \mathrm{pu}$ under full load, resulting in a voltage at the end of the feeder equalling $0.94 \mathrm{pu}$. The conductor segments $Z_{s}$ have a phase angle of 55 degrees (inductive), which is a typical value for overhead lines.

With these considerations, each conductor segment has an impedance of $2.06 \%$ pu (referred to the total rating of the distribution system).

In this section, we have chosen to model an overhead line because the cable capacitance is not negligible in the case of underground cables and therefore, the assumption of a concentrated capacitor would not be correct. In the voltage range of interest (low voltage and lower medium voltage networks), overhead lines appear especially in rural areas.

The linear loads are disconnected to obtain the worst case for the voltage distortion. The placement of the capacitor $\left(X_{C}\right)$ and non-linear load $(N L)$ is not fixed. The non-linear loads are modelled as ideal current sources. For the load current spectrum of the non-linear loads, an $1 / h$ spectrum is assumed for the odd harmonics $(3 \leq h \leq 41)$.

The considered SHI is modelled as a true resistive impedance $Z_{S H I}(h)=R_{s}$ for all harmonics, except for the fundamental component $Z_{S H I}(1)=\infty$.

1) Calculations: For all possible locations of the capacitor and the non-linear load along the feeder, the harmonic mitigation potential of the SHI is investigated for different resistive impedance values ( 0 to $10 \mathrm{pu}$ ) and this for all possible SHI locations along the feeder. For all cases, the relative change of the RMS value of the harmonic voltage components $V_{\text {harm }}$ $\left(V_{\text {harm }}=\sqrt{\sum_{h \neq 1} V_{h}^{2}}\right)$ is calculated and compared with the situation without the SHI $V_{\text {harm, } \infty}$ (i.e. when $Z_{S H I}(h)=$ $\infty, \forall h)$.

In [8], four different network configurations are discussed:

- Capacitor bank and non-linear load located near the beginning of the feeder

- Capacitor bank located near the end of the feeder and non-linear load located near the beginning of the feeder

- Non-linear load and capacitor bank located near the center of the feeder

- Capacitor bank located near the beginning of the feeder and the non-linear load located near the end of the feeder

In the next paragraphs, only the first and the last network configuration will be discussed.

2) Capacitor bank and non-linear load located near the beginning of the feeder: In the first case, both the capacitor bank and the non-linear load are located near the beginning of the feeder. The non-linear loads are concentrated in node 1 (= PCC) and the capacitor is placed in node 3 , as shown in Fig. 4 . The place and the value of the resistive SHI $Z_{S H I}=R_{s}$ 


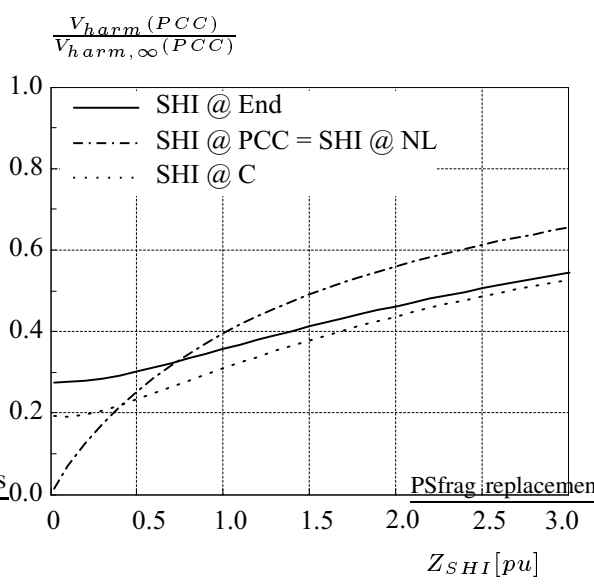

(a) $h_{\text {res }}=3$; Voltage at the PCC, $V_{\text {harm }, \infty}(P C C)=5.36 \%$

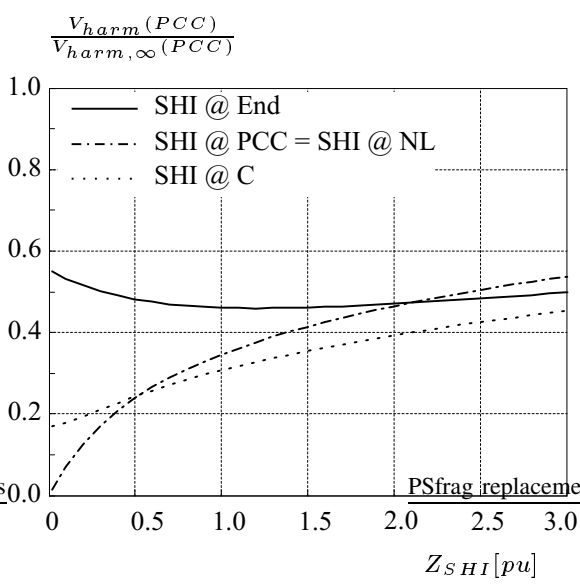

(b) $h_{\text {res }}=11$; Voltage at the PCC, $V_{\text {harm }, \infty}(P C C)=6.14 \%$

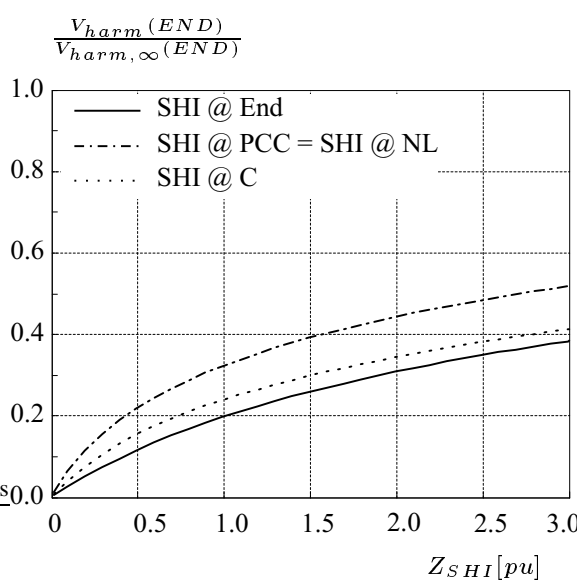

(c) $h_{\text {res }}=11$, Voltage at the END, $V_{\text {harm }, \infty}(E N D)=8.04 \%$

Fig. 5. Relative change of the RMS value of the harmonic voltage components: Capacitor bank (node 3 ) and non-linear load (node 1) located near the beginning of the feeder

is varied. Depending on the value of the capacitor $X_{C}$, different resonance frequencies are obtained. The most important results are shown in Fig. 5. In Fig. 5(a), the reduction of $V_{\text {harm, } \infty}(P C C)$ is shown for a resonance condition at the 3th harmonic order (i.e. when the system impedance $\left|Z_{\text {sys }}(h)\right|$ seen from the non-linear load shows a maximum for the 3 -th harmonic order). For small values of $Z_{S H I}$ (lower than $0.4 \mathrm{pu}$ ), the best place to install the resistive SHI (dashed line) is the PCC. For higher values of $Z_{S H I}$, placing the SHI near the capacitor (dotted line) causes the lowest RMS-value of the harmonic voltage components at the PCC. It is remarkable that placing the SHI at the end of the feeder (solid line) also provides a very good damping of $V_{\text {harm, } \infty}(P C C)$. Remember that values lower than $0.5 p u$ are not encouraged to limit the line current caused by background distortion.

In Fig. 5(b), the reduction of $V_{\text {harm, } \infty}(P C C)$ is shown for a resonance at the 11-th harmonic order. Especially for lower values of $Z_{S H I}$, placing the SHI at the end of the feeder has a less pronounced reduction of $V_{\text {harm, } \infty}(P C C)$ as compared with placing the SHI near the PCC or near the capacitor. The reason is that upon reducing the SHI value, another resonance condition (on a higher harmonic order) is fulfilled, and therefore the reduction of $V_{\text {harm, } \infty}(P C C)$ is quite modest. This is shown in Fig. 6, where the spectrum of the voltage at the PCC is plotted for the 11-th harmonic resonance case. The resonance at the 11-th harmonic order is clearly visible in Fig. 6(b), the case without SHI. Placing the SHI of $1 \mathrm{pu}$ at the end of the feeder (Fig. 6(d)) damps the resonance on the 11-th harmonic order but on the same time, it causes a small resonance on the 13th-harmonic order. That is the reason why the reduction of $V_{\text {harm, } \infty}(P C C)$ is not as pronounced as compared with placing the SHI of 1 pu near the PCC (Fig. 6(c)) or the capacitor.

From Fig. 6(c)-(d), we notice that the lower order harmonic components $(3,5$ and 7 ) are better damped with a 1 pu resistive SHI placed at the end of the feeder than with a harmonic voltage $(\%)$

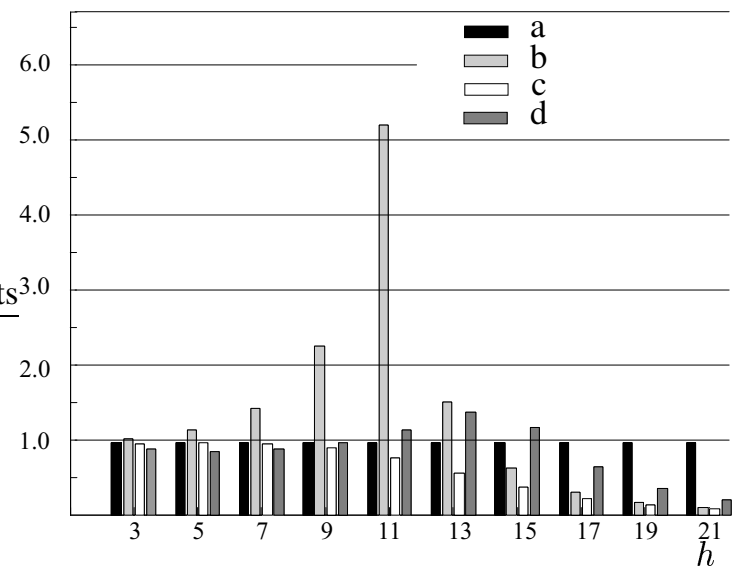

Fig. 6. $h_{r e s}=11$, PCC voltage spectrum when $Z_{S H I}=R_{s}=\infty$, $X_{C}=\infty$ (a), $Z_{S H I}=R_{s}=\infty, X_{C} \neq \infty$ (resonance @ $\left.h=11\right)(\mathrm{b})$, $Z_{S H I}=R_{s}=1 p u$ placed at the PCC (c), $Z_{S H I}=R_{s}=1 p u$ placed at the end of the feeder (d)

1 pu resistive SHI placed at the PCC. On the other hand, the higher harmonic (voltage) components are better damped with a $1 \mathrm{pu}$ resistive SHI placed at the PCC. In practice, higher harmonic components $(h \geq 11)$ of the current are usually smaller than predicted with the $1 / h$ spectrum (e.g. due to attenuation and diversity) and the lower current harmonic components $(\leq 9)$ are generally higher than predicted with the $1 / h$ spectrum. Consequently, the damping performance of a 1 pu resistive SHI placed at the end of the feeder as compared with the damping performance of the same SHI placed at the PCC will be better in practice. This is shown in Fig. 7, where the relative change of the RMS value of the harmonic voltage components at the PCC is shown for a resonance at the 11-th harmonic order with a current spectrum obtained from a time-step simulation of a real rectifier load. In this case, the 1 pu resistive SHI placed at end of the feeder 


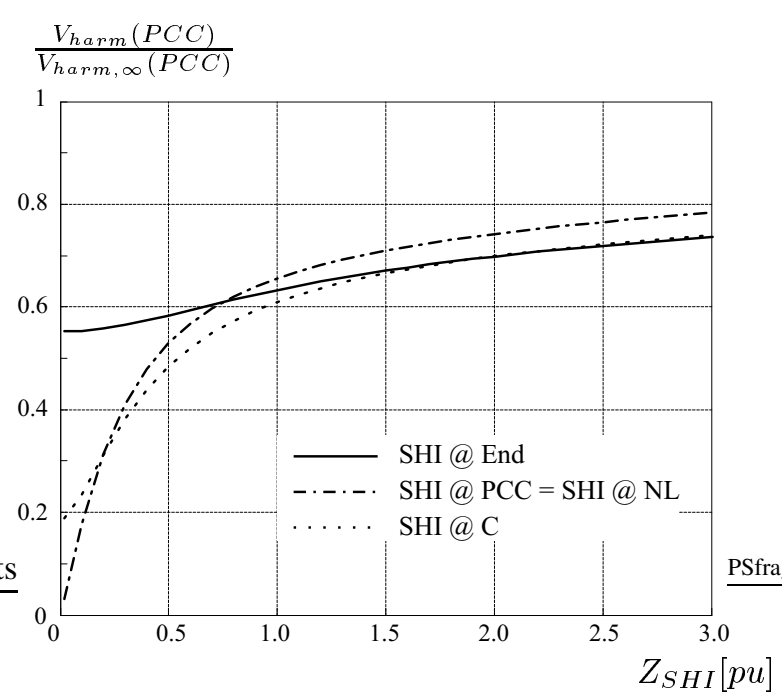

Fig. 7. Relative change of the RMS value of the harmonic voltage components at the PCC for $h_{r e s}=11$, rectifier current spectrum: Capacitor bank (node 3 ) and non-linear load (node 1) located near the beginning of the feeder; $V_{\text {harm }, \infty}(P C C)=4.02 \%$

reduces $V_{\text {harm, } \infty}(P C C)$ more than the same $\mathrm{SHI}$ installed at the PCC. Thus, for a more realistic current spectrum, placing the resistive SHI at the end of the feeder is also in this case a very good choice.

In Fig. 5(c), the relative change of the RMS value of the harmonic voltage components at the end of the feeder is shown for a resonance at the 11-th harmonic order. The reduction of $V_{\text {harm, }, \infty}(P C C)$ is most pronounced when the SHI is placed at the end of the feeder, although the reduction is also large for all other possible SHI locations along the feeder. This is also valid for other resonance conditions and current spectra.

3) Capacitor bank located near the beginning of the feeder and the non-linear load located near the end of the feeder:

In the last case, the capacitor bank is located at the PCC and the non-linear load is placed near the end of the distribution line (node 11).

To reduce $V_{\text {harm, } \infty}(P C C)$, the placement of the resistive SHI is a matter of secondary concern (Fig. 8). However, to reduce $V_{\text {harm, }}(E N D)$, much better results are obtained when the SHI is placed near the end of the feeder (at the end node or at the node of the non-linear load). For the same value of the SHI, the reduction of $V_{\text {harm, } \infty}(E N D)$ (and the resulting damping of the resonance) is at least twice as large for the preferred values of $Z_{S H I}=R_{S H I}$ between 0.5 and $1 \mathrm{pu}$ (Fig.9). The result that the end of the feeder is a good choice to install the SHI remains equally valid for a resonance at the 3-th harmonic order and for the simulations with a rectifier load current spectrum and therefore, the figures are not shown.

4) Summary: It is shown that the THD values of the voltage are highly dependent on the SHI placement along the feeder. However, the end of the feeder is in general not the very best place to install the resistive SHI but it is a good choice to install the SHI, especially when the location of the non-linear load and/or capacitor bank can vary or is unknown. For the two other network configurations, the conclusions are the same but are not discussed in detail in this paper.

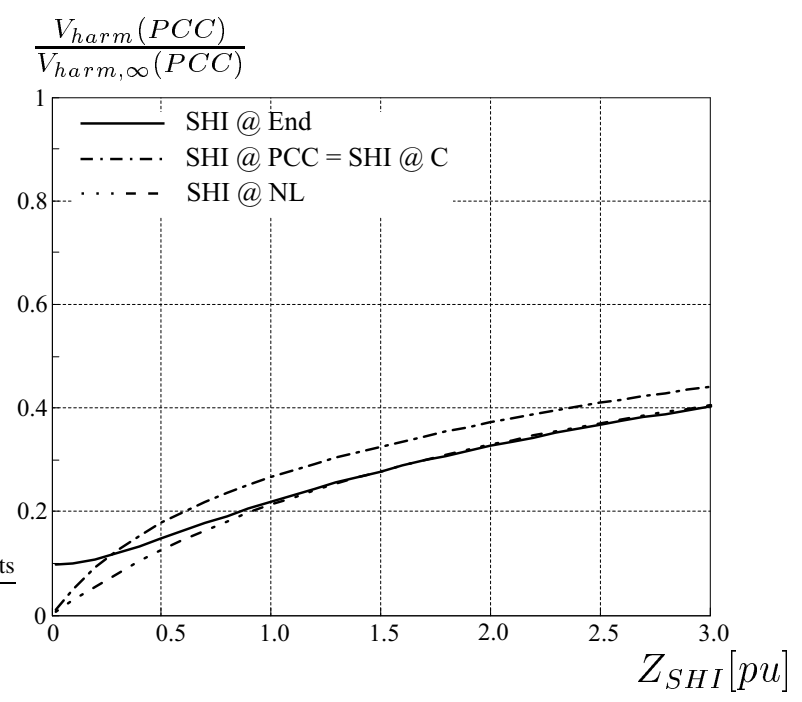

Fig. 8. Relative change of the RMS value of the harmonic voltage components at the PCC for $h_{\text {res }}=11$ : Capacitor bank located near the beginning of the feeder (node 1) and the non-linear load located near the end of the feeder (node 11); Vharm, $\infty$ (PCC) $=8.64 \%$

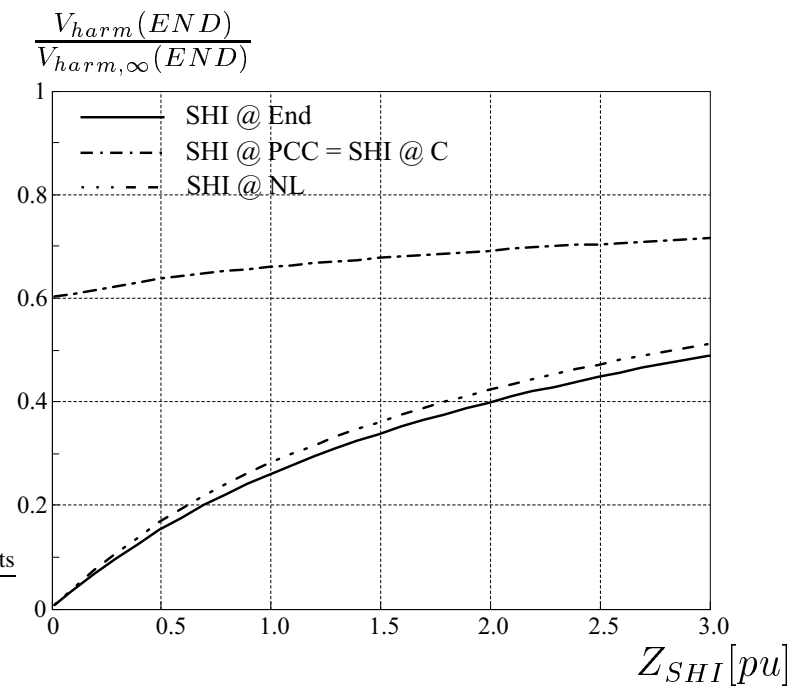

Fig. 9. Relative change of the RMS value of the harmonic voltage components at the END for $h_{r e s}=11$ : Capacitor bank located near the beginning of the feeder (node 1) and the non-linear load located near the end of the feeder (node 11); Vharm, $(P C C)=11.54 \%$

\section{B. Distributed capacitor banks}

At the moment, studies are done to know the influence of distributed capacitors and non-linear loads on the harmonic mitigation potential of a resistive SHI, together with the influence of the SHI-placement on the $T H D_{V}$ reduction. The distributed capacitors have the same value in all nodes and they can model for instance the cable capacitance and/or distributed power factor correction capacitors.

The same distribution network is used as in the previous section but now the conductor segments have a phase angle of 30 degrees (cable network).

The first simulation results confirm the results of the previous section: the end of the feeder is in general a good choice to install the resistive SHI, especially when the system parameters can vary or are unknown. Only with very low resistive SHI values $(\leq 0.2 \mathrm{pu})$, there can occur a harmonic 
magnification of harmonic voltage components in some nodes of the distribution line (Ferranti-effect). This is an extra reason to limit the lower value of the resistive SHI.

In the future, the authors will investigate the application of multiple resistive SHI. In that case, one has to be careful to avoid interaction between different SHI.

\section{Practical Realization}

At the moment, we are dimensioning and building a scale model $\left(S_{r e f}=500 \mathrm{VA}\right)$ of a distribution system. The system setup is the same as in Fig. 4, but with 3 phases and a neutral conductor. The capacitor value in each node is variable to obtain different resonance conditions. The phase angle of the conductor segments can vary to model underground cables as well as overhead lines. Together with a $1 k V A$ voltage source, this system setup can be used to check all simulation results. In the near future, there will be started with the practical realization of the resistive impedance as a secondary control function of a power electronic energy supply. To realize the function $v_{a, b, c}=K_{v} \cdot i_{a, b, c}\left(K_{v}=\right.$ real $)$, one needs the measurements of the mains voltage $v_{a, b, c}$ and $v_{D C}$ [9]. With these measurements, the PWM signals can be generated (with a DSP) to obtain the resistive behaviour for harmonics (Fig.10), and that, eventually, with a different resistive value for different harmonic orders. Moreover, there are no restrictions for the fundamental component of the current, e.g. it can be capacitive to generate reactive power.

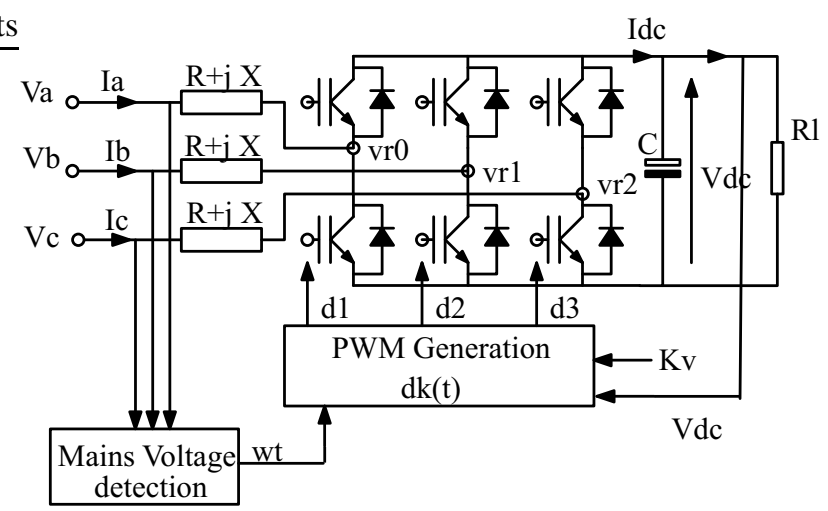

Fig. 10. Practical realization of the SHI

\section{ACKNOWLEDGMENTS}

The research of Wouter Ryckaert is supported by the Institute for the Promotion of Innovation by Science and Technology in Flanders (IWT).

\section{REFERENCES}

[1] IEEE Task Force on the effect of harmonics on equipment, Effects of harmonics on equipment, IEEE Transactions on Power Delivery 8 (2)(1993) 672-680.

[2] F.Z. Peng, Application issues of active power filters, IEEE Industry Applications Magazine 4 (5) (1998) 21-30.

[3] H.Akagi, New trends in active power line conditioners, IEEE Transactions on Industry Applications 32 (6) (1996) 1312-1322.

[4] H.Akagi, H. Fujita, and K. Wada, A shunt active filter based on voltage detection for harmonic termination of a radial power distribution line, IEEE Transactions on Industry Applications 35 (3) (1999) 638-645.
[5] D.M. Van de Sype, K. De Gussemé, A.P. Van den Bossche, and J.A.A. Melkebeek, Duty-ratio feedforward for digitally controlled boost PFC converters, Proceedings of the IEEE-Appl. Power Electr. Conf., APEC 2003, Feb. 9-13, 2003, Miami, USA, pp. 396-402

[6] W. Ryckaert, J. Ghijselen, and J. Melkebeek, Harmonic Mitigation Potential of Shunt Harmonic Impedances and the Influence of Background Distortion, Proceedings of the IEEE PES Power Tech Conference, ISBN 0-7803-7968-3, Bologna, Italy, 23-26 June 2003.

[7] W. Ryckaert, J. Ghijselen, and J. Melkebeek, Harmonic Mitigation Potential of Shunt Harmonic Impedances, Electric Power Systems Research 65 (3) (2003) 63-69.

[8] W. Ryckaert, J. Ghijselen, and J. Melkebeek, Harmonic Mitigation Potential of a Resistive Shunt Harmonic Impedance in Correlation with its Placement along a Distribution Feeder. Proceedings of the CIGRE/IEEE PES international symposium on Quality and Security of Electric Power Delivery Systems, ISBN 2-85873, Montréal, Canada, 8-10 October 2003.

[9] J. Ghijselen, Actieve gelijkrichters voor optimale vermogenskwaliteit $\mathrm{Ph} . \mathrm{D}$. dissertation, Ghent University, Faculty of Engineering, Ghent, Belgium, Nov. 2001 1416-1422. 